\title{
GERD and idiopathic pulmonary fibrosis: cause or effect
}

\author{
Stephenie M. Takahashi $•$ Imre Noth
}

Published online: 30 October 2013

(C) Springer Science+Business Media New York 2013

\begin{abstract}
Idiopathic pulmonary fibrosis is a chronic fibrosing disease of the lung and is the most common of the interstitial pneumonias. The pathophysiology of IPF involves recurrent epithelial cell injury, abnormal wound repair response and aberrant fibroblast proliferation. Recent studies have provided information regarding the potential function of gastroesphageal reflux (GER) and microaspiration as a possible etiology of this recurrent epithelial injury and fibrosis formation. Although acid aspiration has been linked to IPF, the mechanism involved and the relationship of abnormal GER to the cause or progression of IPF have not been adequately addressed. This association is of great interest to the scientific and clinical communities, with ongoing active investigations. Herein, we review current understanding about the relationship between GER and IPF, and investigate the evidence supporting this relationship.
\end{abstract}

Keywords Gastroesophageal reflux · Interstitial lung disease · Idiopathic pulmonary fibrosis $\cdot$ Microaspiration $\cdot$ Hiatal hernia

$\begin{array}{ll}\text { Abbreviations } \\ \text { IPF } & \text { Idiopathic pulmonary fibrosis } \\ \text { GER } & \text { Gastroesophageal reflux } \\ \text { GERD } & \text { Gastroesophageal reflux disease } \\ \text { LES } & \text { Lower esophageal sphincter } \\ \text { COPD } & \text { Chronic obstructive pulmonary disease } \\ \text { PPI } & \text { Proton-pump inhibitor } \\ \text { EBC } & \text { Exhaled breath condensate }\end{array}$

S. M. Takahashi $(\bowtie) \cdot$ I. Noth

The University of Chicago Medical Center, 5841 S. Maryland

Avenue, MC 6076, Chicago, IL 60637, USA

e-mail: Stephenie.Takahashi@uchospitals.edu

I. Noth

e-mail: inoth@medicine.bsd.uchicago.edu
BAL Bronchioalveolar lavage

ILD Interstitial lung disease

CT Computed Tomography

NSIP Non-specific interstitial pneumonitis

PFT Pulmonary function test

DLCO Diffusing capacity of the lung for carbon monoxide

TLC Total lung capacity

FVC Forced vital capacity

OSA Obstructive sleep apnea

\section{Introduction}

Idiopathic pulmonary fibrosis (IPF) is a chronic fibrosing disease of the lung and is the most common of the interstitial pneumonias. To date there is no approved therapy for this disease, which has a $2-5$ year $50 \%$ mortality [1]. The pathophysiology of IPF is believed to be based on recurrent epithelial cell injury and abnormal wound repair responses, including aberrant fibroblast activity [2]. The cause of epithelial injury remains unknown, but cigarette smoking, chronic viral infections, exposure to wood and dust particles, and drug toxicity have been associated with IPF [3]. In addition to these exposures, recent studies have revealed the potential role of gastroesphageal reflux (GER) and microaspiration as a possible cause of recurrent epithelial injury [4]. GER includes reflux of both acidic and non-acidic content, symptomatic disease, and asymptomatic events. However, although a high incidence of acid aspiration in IPF has been detected in several series, the role of GER in either the cause or the progression of IPF is not well understood [5•], and the association of GER and IPF is a topic of intense ongoing investigation. Herein, we review the data on GER in IPF, and discuss the implications of this association regarding screening for and treating of GER in patients with IPF. 


\section{Pathophysiology and diagnosis of gastroesophageal reflux disease}

Gastroesophageal reflux disease (GERD) is defined as reflux of gastric contents into the esophagus, leading to symptomatic esophagitis, reflux symptoms sufficient to impair quality of life, or an increased risk of long-term complications [6]. This definition emphasizes that GER becomes a disease (GERD) when it affects quality of life or when it causes macroscopic damage to the esophagus [7]. GERD, when defined by weekly symptoms and/or acid regurgitation, is very common, affecting 10-20\% of people in the Western world [8]. GER has a variety of possible causes, and the association between age and development of reflux is unclear.

GER is believed to occur as a result of the failure of normal protective physiological mechanisms. These protective mechanisms include the combined action of the lower esophageal sphincter (LES), a segment of smooth muscle that contracts to a pressure greater than that of the stomach, and the crural diaphragm, which provides extrinsic pressure and a barrier separating the esophageal and gastric compartments [9]. The pathophysiology of reflux is believed to be caused by impaired function of the LES in particular, because contraction of this smooth muscle serves as a barrier preventing gastric contents entering the lower esophagus [10]. Dysfunction of the LES can be mechanical, resulting from loss of tone, or functional, where the frequency of relaxation is increased. Although temporary relaxations of the LES are normal, these episodes are believed to occur more frequently in individuals with GERD, and can result in prolonged periods of exposure of refluxed gastric contents [11].

The pressure gradient between the abdomen and thorax can also affect the development of GER. It has been postulated that patients with chronic obstructive pulmonary disease (COPD), who have increased negative intra-thoracic pressure, increased lung compliance, and reduced elastic recoil, may be predisposed to reflux $[12,13]$. Indeed, a higher prevalence of diaphragmatic defects and hiatal hernias was documented in patients with emphysema [14]. In addition, it has been postulated that restrictive lung disease can affect the pressure in the intrathoracic space, reducing the overall lung volume, which can disrupt the diaphragm and potentially increase reflux.

The presence of a hiatal hernia increases the time of LES relaxation, and increases the risk of esophagitis, GER, and GERD [15]. Hiatal hernias alter the crux of the diaphragm, which can structurally alter the LES. Given this, hiatal hernia has been associated with episodes of microaspiration, diminished pressure of the LES, and increased risk of erosive esophagitis [16].

Because GER can occur without GERD, the presence of symptoms is a poor indicator of underlying reflux and aspiration. This may be particularly true for patients with IPF [4]. Therefore, diagnostic studies are often necessary to adequately assess for aspiration. Commonly used diagnostic approaches for GER include a proton-pump inhibitor (PPI) trial, endoscopy, or 24-hour $\mathrm{pH}$ monitoring with or without esophageal manometry [7]. Less common diagnostic tests include gastroesophageal-pulmonary scintigraphy, testing for pepsin and bile salts in bronchoalveolar lavage fluid (BAL), and testing of exhaled breath condensate (EBC) [4]. Ambulatory $\mathrm{pH}$ monitoring measures the $\mathrm{pH}$ at two different esophageal sites, generating a composite $\mathrm{pH}$ score (the DeMeester score); therefore, $\mathrm{pH}$ monitoring is regarded as more sensitive and specific for regurgitation [17]. Overall, given the limitations of any individual test, clinicians frequently use a combination of these tests to diagnose GER.

\section{Pulmonary manifestations of GER}

If a patient suffers from symptomatic or asymptomatic GER, they may or may not have pulmonary manifestations of disease. Not all patients with GER or GERD have aspiration. However, if the gastric contents travel as high as the cricopharyngeal region, esophageal contents can enter the airway. The inhalation of oropharyngeal or gastric contents into the larynx or lower respiratory tract is defined as aspiration [18] (Fig. 1). The pulmonary syndrome resulting from an aspiration event depends on the content of the material, the volume of aspirate, and the response of the host. Asymptomatic aspiration of oropharyngeal secretion or gastric fluid into the lungs is called silent microaspiration. It has been demonstrated that approximately half of normal adults experience mild silent microaspiration during sleep at night [19]. Normally, natural defenses, including coughing and epiglottic closure, are protective against microaspiration. Depending on the individual, sometimes these defenses become impaired and cause wheezing and coughing [20].

There is increasing recognition that gastric refluxate, either acidic or nonacidic, can be a risk factor for many respiratory symptoms, including chronic cough [21,22] and hoarseness [23]. In both animal and clinical data, it has been observed that GER may evoke bronchospasm and/or potentiate the bronchomotor response to additional triggers [24]. The correlation between GER and asthma has been observed by multiple studies, revealing that asthma patients have a higher incidence of GER symptoms, esophagitis, and hiatal hernias [25, 26]. Asthmatics with reflux also had a significant dose-response association between breathlessness and reflux symptoms [27].

It has been reported that $71 \%$ of asthmatic patients with GER symptoms benefited from medical therapy or lifestyle modification, as assessed by measurement of improved pulmonary symptoms [28]. As well as asthma, many other respiratory disorders have been implicated, including COPD, bronchiectasis, chronic bronchitis and pneumonia [29]. Although these associations have been established, it is 


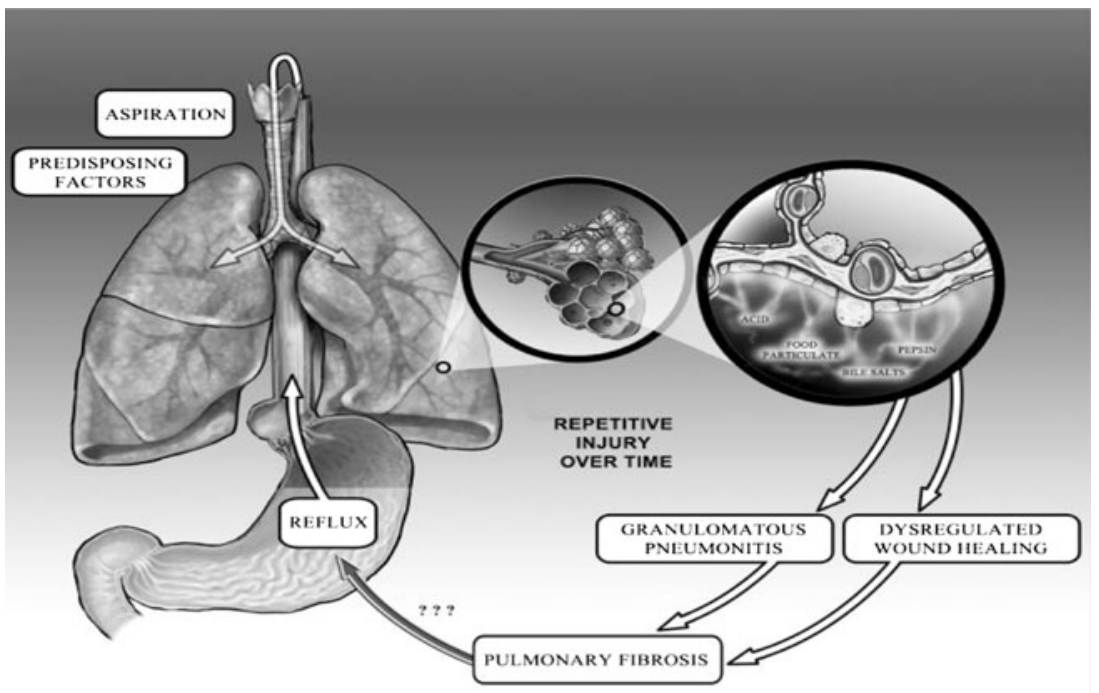

Fig. 1 Potential mechanism of chronic aspiration in IPF. Gastric contents can travel through a weakened lower esophageal sphincter up into the esophagus. If the gastric contents travel as high as the cricopharyngeal region, esophageal contents can enter the airway. Chronic microaspiration may cause repeated injury, leading to dysregulated wound healing, granulomatous pneumonitis, and lung fibrosis. Reprinted from the American Journal of Medicine, Vol 123, Lee JS et al., Chronic Microaspiration and Idiopathic Pulmonary Fibrosis, 304-11, Copyright (2010), with permission from Elsevier difficult to determine a definitive causal relationship between GER and lung disease. Given this, many hypothesize that repetitive injury, from either acidic or non-acidic reflux into the lungs, can lead to epithelial cell injury and fibrosis.

The potential relevance of GER to pulmonary fibrosis is highlighted by scleroderma outcomes. Over $50 \%$ of patients with scleroderma develop interstitial lung disease (ILD), with fibrotic nonspecific interstitial pneumonitis (NSIP) the most commonly observed histopathological pattern [30, 31]. Esophageal dysfunction, a very common complication of scleroderma, is marked by reduced LES pressure, which is a strong risk factor for reflux events, and reduced peristalsis, which leads to prolonged refluxate exposure.

In scleroderma, the relationship between esophageal dysfunction and ILD has been a topic of active investigation, but remains unknown. In earlier studies, no definite association was observed between GER and pulmonary function $[32,33]$. Since then, prospective studies have added to our understanding of the relationship between GER and ILD in scleroderma. Marie et al. found that esophageal dysfunction, determined by use of manometry, was associated with a higher prevalence of ILD on CT imaging. Furthermore, the prevalence of ILD increased with increasing severity of esophageal dysfunction [34]. In addition, patients with severe esophageal dysfunction had a significant reduction of diffusing capacity of the lung for carbon monoxide (DLCO) compared with patients with no or mild esophageal dysfunction. More recently, Savarino et al. found that patients with scleroderma complicated by ILD had reduced LES pressures and an increased frequency of proximal reflux events compared with scleroderma patients without ILD [35]. They revealed a dose-response association, with reflux frequency positively correlated with radiographic fibrosis scores. Proximal esophageal reflux was also observed, suggesting that GER in scleroderma may indeed lead to microaspiration. Although the results from these studies suggest an association between GER and ILD, causality remains to be proved. Gilson et al., in their prospective evaluation, were not able to establish that esophageal dysfunction was a significant predictor of PFT decline [36•]. In short, although several studies suggest an association between esophageal dysfunction and ILD in scleroderma, varying methods have lead to conflicting reports, and further study is needed.

\section{GER in IPF patients}

There is long-standing interest, dating back over fifty years, in the function of repetitive microaspiration in the development of IPF. During the last two decades, several studies have investigated the prevalence and incidence of GER, and the effect of GER treatment, in patients with IPF. In one of the first clinical reports on GER and lung injury, Tobin and colleagues evaluated the prevalence of acid reflux in patients with IPF compared with control patients with ILD. In this prospective study, ambulatory $\mathrm{pH}$ monitoring to diagnose GER was performed on 17 consecutive newly diagnosed IPF patients and on eight control patients with ILD. GER was significantly more prevalent in the IPF group [37]. Ninety-four percent of 
IPF patients had abnormal distal or proximal esophageal acid exposure, compared with $50 \%$ of control ILD patients. Interestingly, most of the IPF patients with abnormal esophageal reflux were asymptomatic, and there was no correlation between DLCO and acid exposure. The IPF patients had a particularly high incidence of proximal acid exposure in the supine or nocturnal position, which is very uncommon in healthy individuals. The authors hypothesize that there are increased GER events during sleep, when the upper esophageal sphincter pressure is greatly reduced and cough reflexes are suppressed. This increased GER may lead to increased repetitive microaspiration events, and may be predictive of potential repetitive injury for IPF patients.

Later in a large prospective study, the investigators examined 65 IPF patients and 133 asthma patients; the prevalence of abnormal acid reflux in the IPF patients was $87 \%$, significantly higher than in patients with asthma. They found that half the IPF patients were asymptomatic and also found no correlation of GER severity with pulmonary function, supporting previous results [38]. Because $65 \%$ of the IPF patients were taking PPIs, and because asthmatics were used as controls, the prevalence of abnormal acid reflux may have been underestimated. This study was the first to introduce the indication for ambulatory $\mathrm{pH}$ monitoring for all IPF patients, whether symptomatic or asymptomatic. Twelve of the 19 patients receiving therapy for acid suppression had abnormal acid exposure revealed by $\mathrm{pH}$ monitoring, suggesting that standard doses of PPIs may not be effective management for IPF patients.

Bandeira and colleagues also attempted to quantify the prevalence of abnormal acid reflux in IPF patients by use of multiple diagnostic tests [39]. In agreement with Tobin's work, they found that a substantial percentage $(80 \%)$ of these patients experience reflux events in the supine position. There was no association between pulmonary function and GER. The authors note that the IPF patients were at different stages of diagnosis and disease progression during this study, making it difficult to assess any causality of this association.

Similar to the procedure for non-ILD patients, diagnosis of GERD in IPF is often made by use of a combination of different diagnostic tools. In a recent study, GER in IPF patients was evaluated by use of a reflux cough questionnaire, measurement of pepsin in the exhaled breath condensate (EBC), and H. pylori antibody detection by use of ELISA [40•]. EBC measurement is believed to be a reliable technique for measuring pepsin as a surrogate for GER in the upper airways at the time of reflux episodes. Additionally, the relationship between pulmonary microaspiration and pepsin concentration in bronchoalveolar lavage fluid has been demonstrated in animal and human studies. Although EBC pepsin was detected for two out of 17 IPF patients vs. none of the controls, the difference was not significant. In addition, there was no significant difference in $H$. pylori serology for
IPF patients vs. controls. Although a small study, the reflex cough questionnaire score - a validated measure of non-acidic airway reflux - was significantly higher for IPF patients, revealing the potential importance of a diagnosis of nonacidic reflux disease for these patients. Recently, more studies have addressed asymptomatic GER and the choice of diagnostic test. A recent prospective study of patients with ILD and IPF reestablished that the sensitivity and specificity of symptoms alone was very low and that esophageal function testing should be used to establish a diagnosis of GER for these patients [41•].

By investigating GER in IPF patients referred for lung transplantation, potentially a more severely ill group of patients, more insight has been gained. In a study by Sweet and colleagues, $67 \%$ of IPF patients referred for lung transplantation had abnormal esophageal reflux, and $65 \%$ of these patients had a hypotensive LES. Furthermore, $50 \%$ of patients with reflux had abnormal esophageal peristalsis [42]. The authors note that distal reflux and markers of microaspiration have been associated with early chronic allograft rejection after transplantation, suggesting microaspiration may cause direct lung injury [43, 44]. In addition to verifying a high prevalence of GER in IPF, this study also confirmed the finding that symptoms do not significantly correlate with reflux severity.

Elevated pepsin in BAL samples has proved a useful biomarker for aspiration in lung transplant recipients [45]. It has been previously hypothesized that acute exacerbations of IPF may be caused by microaspiration [46]. Pepsin levels in BAL fluid from patients experiencing exacerbations of IPF were significantly elevated compared to levels of control patients with stable disease [47•]. It remains to be determined whether pepsin levels differ, and if so by how much, for stable patients with IPF or patients in the early stages of disease compared with healthy controls.

An asymmetrical fibrotic pattern on computed tomography (CT) scans may also be a potential indicator of microaspiration. Symptomatic reflux and GER on objective testing were higher for 32 IPF patients with more than $20 \%$ asymmetrical fibrosis by CT scan, than for those without asymmetrical fibrosis $(62.5$ vs. $31.3 \%$ ). Furthermore, patients with asymmetrical disease had reduced DLCO values compared with controls [48•]. In addition, the patients with asymmetrical disease had a higher incidence of acute exacerbations of IPF (46.9 vs. $17.2 \%$ ), and the right side of the lung was more involved. These results not only support the effect of GER in the etiology of acute exacerbation of IPF, but also introduce another modality, identification of an asymmetrical pattern on CT scan, to help identify and diagnose IPF patients with GER.

Increased prevalence of obstructive sleep apnea has been observed in IPF patients, which may affect development of GER. Swigris and colleagues examined 54 subjects with fibrosing ILD, who had undergone a polysomnogram and 
$\mathrm{pH}$ monitoring, and used logistic regression to determine independent predictors. They found that neither OSA nor the apnea hypopnea index (AHI) predicted the presence of GER. And, regardless of body position, GER was no more frequent in those with OSA than those without OSA [49•].

Hiatal hernia has a known association with GER and may contribute to the development and/or severity of IPF. It is believed that the presence of a hiatal hernia contributes to weakness of the LES and is therefore associated with GER and esophagitis [16, 50]. In respiratory disorders, it is unclear how hiatal hernia may contribute to or affect the nature of pressure variations in the thorax. Obstructive lung disease can lead to increased thoracic pressure, which may predispose to development of hiatal hernia by pushing down on the diaphragm. In the case of restriction, volume loss may lead to pulling up on the diaphragm, resulting in disruption of the integrity of the LES sphincter. Noth and colleagues retrospectively examined the incidence of hiatal hernia in IPF patients compared with asthma or COPD patients [5•]. The prevalence of hiatal hernia, diagnosed by thoracic radiologists from CT scans, was significantly higher in IPF patients (39\%) compared with asthma or COPD controls (16.7 \% and $13.3 \%$, respectively). Although there were no differences in pulmonary function or composite physiological index (CPI) for IPF patients with and without hiatal hernia, IPF patients with hiatal hernia who were on anti-reflux therapy had significantly higher percent-predicted DLCO than those not on therapy (58\% versus $50 \%$ ). A small cohort of these patients underwent esophageal $\mathrm{pH}$ monitoring and manometry, which revealed that patients with hiatal hernia had significantly higher DeMeester scores (22.8) than those without hiatal hernia (10.2). Because asthma and COPD patients have an increased incidence of hiatal hernia, using them as control patients suggests an even higher incidence of hiatal hernia for IPF patients. Although previous studies were unable to associate decline in lung function with presence of reflux, this study indicates that treatment of IPF patients with hiatal hernia may have a protective effect on lung function.

The many clinical studies performed to date identify an increased prevalence of GER in IPF. A variety of diagnostic modalities have been used, often in combination, to establish GER in IPF. These include assessing for symptoms, ambulatory esophageal $\mathrm{pH}$ monitoring, esophageal manometry, EBC, and the presence of hiatal hernia. Because many studies suggest that GER in IPF patients may be asymptomatic, clinicians are encouraged to have a low threshold for evaluation for GER.

\section{Treatment of GERD in IPF}

IPF is a relentless, progressive disease that is nearly always fatal. There are currently no approved medications for patients with this disease. Historically, anti-fibrotic or antiinflammatory therapy has proved ineffective at altering disease progression or improving survival. Therefore the concept of treating reflux disease in IPF is appealing to pulmonary care providers and to researchers of IPF. However, because of the risks associated with medical or surgical treatment of reflux disease, pulmonary clinicians are unsure of how aggressive treatment should be.

Several studies have examined whether treating GERD, either medically or surgically, can affect pulmonary symptoms and fibrosis progression. Raghu and colleagues assessed whether acid suppression treatment alone would prevent progression of disease for IPF patients. In a retrospective review, four newly diagnosed IPF patients with documented increased GER were treated with PPIs and Nissen fundoplications for repair of hiatal hernia (if present) [13]. Two of the four patients deteriorated with non-adherence to anti-acid therapy, but subsequently stabilized with re-initiation of therapy. Although the clinical courses were variable, and although the numbers were small, the authors concluded that treatment of GER was associated with stabilization or improvement of respiratory status, as measured by FVC and DLCO. During the follow-up period, none of the patients required treatment for respiratory decompensation, and in particular none experienced an acute exacerbation of IPF. The authors suggest that suppression of acid and preventing GER in those who underwent surgery for hiatal hernia halted progression of fibrosis. These findings were regarded as supporting the hypothesis that repetitive parenchymal injury is mediated by chronic microaspiration of acid. Although limited by its retrospective nature, and although no definitive conclusions can be drawn from such a small cohort, this study was the first to establish that treatment of GER may be beneficial for halting the progression of pulmonary fibrosis.

As mentioned previously, the presence of reflux in the context of lung transplantation might predispose individuals to develop bronchiolitis obliterans. Linden and colleagues compared 14 IPF patients awaiting lung transplant who underwent laproscopic Nissen fundoplications with IPF patients who did not undergo surgery [51]. There were no complications from surgery, and the authors concluded that such laproscopic procedures could be performed safely for patients with advanced IPF. Post-surgery, no patients had recurrent heartburn or regurgitation symptoms, although some experienced superficial wound infections and esophageal strictures. Furthermore, surgery was associated with a reduction in supplemental oxygen, from $3.0 \mathrm{~L} \mathrm{~min}^{-1}$ to $2.5 \mathrm{~L} \mathrm{~min}^{-1}$, whereas those who did not undergo surgery had an average increase from $2.0 \mathrm{~L} \mathrm{~min}^{-1}$ to $3.0 \mathrm{~L} \mathrm{~min}^{-1}$. In addition, over a mean follow-up of 15 months, exercise capacity and lung function remained stable for the surgical treatment group. Although these results are encouraging, this was a relatively small retrospective study. Larger randomized trials of surgical 
intervention for IPF patients with reflux will be informative, and are in progress (UO1 UCSF Collard is lead PI).

A recent large study of over 200 IPF patients examined the survival of patients with reflux disease [52••]. A regression analysis found the use of GERD medication to be an independent predictor of longer survival. Furthermore, use of GERD medication was associated with a lower radiographic fibrosis score. Although these results suggest that treatment of GERD may confer a survival benefit, it is possible that nonrandomized patients receiving GERD therapy may also receive other valuable interventions, for example pulmonary rehabilitation and pneumonia vaccinations. Moreover, patients who have GERD and are on medication may have been diagnosed with IPF sooner than others, leading to earlier treatment. Recently, IPF patients included in three randomized controlled trials were analyzed for changes to pulmonary function while taking GERD medication. Of the 242 patients, patients taking anti-acid therapy at baseline had a smaller reduction in their forced vital capacity (FVC) than those not taking therapy [53••], again revealing that therapy could be beneficial and aid in stopping disease progression.

Cumulatively, these studies suggest that medical and/or surgical therapy for GER may have a positive effect on important clinical variables for IPF, including supplemental oxygen requirement, progression of pulmonary function, and perhaps even survival. Because of the current lack of approved anti-fibrotic therapy for IPF, and the relative ease of treating reflux disease, many clinicians attempt empirical GER treatment. Further investigation is needed to more definitively clarify the role of GER treatment in the care of IPF. The results of studies evaluating anti-reflux surgical interventions for IPF will inform our understanding of the pathogenic function of duodenogastric reflux, where surgery is a more definitive therapy for bile acid reflux. Because the production of biliary secretions may not be affected by PPI therapy, the distinction between duodenogastric reflux and gastric reflux has therapeutic implications.

\section{Summary}

Multiple investigations have established an association between GER and IPF, and reflux has been implicated as having a potential function in the etiology and development of IPF. However, although GER may be highly prevalent among IPF patients, it is unclear how microaspiration events contribute to lung function decline and how to accurately measure these events. More accurate diagnostic measures for microaspiration are needed to advance our understanding of the effect of aspiration and fibrotic damage. In particular, it is still uncertain whether GER causes fibrosis, or fibrosis causes reflux. The former implies that repetitive injury caused by microaspiration, acidic or non-acidic, may be a source of epithelial damage, which in a genetically susceptible IPF host may result in poor wound healing and an exaggerated fibroproliferative response. Regarding the latter, some hypothesize that pulmonary fibrosis results in altered respiratory mechanics that can subsequently induce associated reflux disease. In particular, reduced lung compliance leads to increased negative intrapleural pressures, which can be transmitted to other mediastinal structures including the LES. Previously, many smaller studies have not revealed a relationship between extent of lung function decline and the presence of reflux disease. We do not know whether treating microaspiration will affect the course of IPF. Until a causal relationship is established and the effect of therapy demonstrated, the extent to which IPF patients should receive treatment for asymptomatic GER and GERD remains unknown. Further research into the pathophysiology of IPF and the response to reflex therapeutic interventions is ongoing. These efforts will no doubt improve our care of patients with this devastating disease.

\section{Compliance with Ethics Guidelines}

Conflict of Interest Stephenie M. Takahashi declares that she has no conflicts of interest.

Imre Noth is a paid board member for BI and a paid consultant for Immuneworks. He receives money for expert testimony, and his institution receives money through grants from Sanofi, Stromedix, and NIH.

Human and Animal Rights and Informed Consent All studies by the authors involving human subjects were performed after approval by the appropriate Institutional Review Boards. Written informed consent was obtained from all participants.

\section{References}

Papers of particular interest, published recently, have been highlighted as:

- Of importance

•• Of major importance

1. Raghu G, Collard HR, Egan JJ, Martinez FJ, Behr J, Brown KK, et al. An official ATS/ERS/JRS/ALAT statement: idiopathic pulmonary fibrosis: evidence-based guidelines for diagnosis and management. Am J Respir Crit Care Med. 2011;183(6):788-824.

2. Harari S, Caminati A. Idiopathic pulmonary fibrosis. Allergy. 2005;60(4):421-35.

3. American Thoracic Society. Idiopathic pulmonary fibrosis: diagnosis and treatment. International consensus statement. American Thoracic Society (ATS), and the European Respiratory Society (ERS). Am J Respir Crit Care Med. 2000;161(2 Pt 1):646-64.

4. Lee JS, Collard HR, Raghu G, Sweet MP, Hays SR, Campos GM, et al. Does chronic microaspiration cause idiopathic pulmonary fibrosis? Am J Med. 2010;123(4):304-11.

5. • Noth I, Zangan SM, Soares RV, Forsythe A, Demchuk C, Takahashi $\mathrm{SM}$, et al. Prevalence of hiatal hernia by blinded multidetector CT in patients with idiopathic pulmonary fibrosis. Eur Respir J. 2012;39(2): 
344-51. This study was the first to identify increased prevalence of hiatal hernia, diagnosed by thoracic radiologists on CTscans, in IPF patients compared with asthma or COPD controls.

6. Dent J, Armstrong D, Delaney B, Moayyedi P, Talley NJ, Vakil N. Symptom evaluation in reflux disease: workshop background, processes, terminology, recommendations, and discussion outputs. Gut. 2004;53 Suppl 4:iv1-24.

7. Moayyedi P, Talley NJ. Gastro-oesophageal reflux disease. Lancet. 2006;367(9528):2086-100.

8. Dent J, El-Serag HB, Wallander MA, Johansson S. Epidemiology of gastro-oesophageal reflux disease: a systematic review. Gut. 2005;54(5):710-7.

9. Fahim A, Crooks M, Hart SP. Gastroesophageal reflux and idiopathic pulmonary fibrosis: a review. Pulm Med. 2011;2011:634613.

10. Kahrilas PJ. GERD pathogenesis, pathophysiology, and clinical manifestations. Cleve Clin J Med. 2003;70 Suppl 5:S4-19.

11. Dent J. Patterns of lower esophageal sphincter function associated with gastroesophageal reflux. Am J Med. 1997;103 (5A):29S-32.

12. Demeter P, Pap A. The relationship between gastroesophageal reflux disease and obstructive sleep apnea. J Gastroenterol. 2004;39(9): 815-20.

13. Raghu G, Yang ST, Spada C, Hayes J, Pellegrini CA. Sole treatment of acid gastroesophageal reflux in idiopathic pulmonary fibrosis: a case series. Chest. 2006;129(3):794-800.

14. Caskey CI, Zerhouni EA, Fishman EK, Rahmouni AD. Aging of the diaphragm: a CT study. Radiology. 1989;171(2):385-9.

15. Jones MP, Sloan SS, Rabine JC, Ebert CC, Huang CF, Kahrilas PJ. Hiatal hernia size is the dominant determinant of esophagitis presence and severity in gastroesophageal reflux disease. Am J Gastroenterol. 2001;96(6):1711-7.

16. Lord RV, DeMeester SR, Peters JH, Hagen JA, Elyssnia D, Sheth CT, et al. Hiatal hernia, lower esophageal sphincter incompetence, and effectiveness of Nissen fundoplication in the spectrum of gastroesophageal reflux disease. J Gastrointest Surg. 2009;13(4): $602-10$.

17. Johnson LF, Demeester TR. Twenty-four-hour $\mathrm{pH}$ monitoring of the distal esophagus. A quantitative measure of gastroesophageal reflux. Am J Gastroenterol. 1974;62(4):325-32.

18. Marik PE. Aspiration pneumonitis and aspiration pneumonia. N Engl J Med. 2001;344(9):665-71.

19. Vaezi MF, Singh S, Richter JE. Role of acid and duodenogastric reflux in esophageal mucosal injury: a review of animal and human studies. Gastroenterology. 1995;108(6):1897-907.

20. Warner MA, Warner ME, Weber JG. Clinical significance of pulmonary aspiration during the perioperative period. Anesthesiology. 1993;78(1):56-62.

21. Kastelik JA, Redington AE, Aziz I, Buckton GK, Smith CM, Dakkak $\mathrm{M}$, et al. Abnormal oesophageal motility in patients with chronic cough. Thorax. 2003;58(8):699-702.

22. Ford AC, Forman D, Moayyedi P, Morice AH. Cough in the community: a cross sectional survey and the relationship to gastrointestinal symptoms. Thorax. 2006;61(11):975-9.

23. Wiener GJ, Koufman JA, Wu WC, Cooper JB, Richter JE, Castell DO. Chronic hoarseness secondary to gastroesophageal reflux disease: documentation with 24-h ambulatory $\mathrm{pH}$ monitoring. Am J Gastroenterol. 1989;84(12):1503-8.

24. Simpson WG. Gastroesophageal reflux disease and asthma. Diagnosis and management. Arch Intern Med. 1995;155(8):798803.

25. Harding SM, Guzzo MR, Richter JE. The prevalence of gastroesophageal reflux in asthma patients without reflux symptoms. Am J Respir Crit Care Med. 2000;162(1):34-9.

26. Havemann BD, Henderson CA, El-Serag HB. The association between gastro-oesophageal reflux disease and asthma: a systematic review. Gut. 2007;56(12):1654-64.
27. Nordenstedt H, Nilsson M, Johansson S, Wallander MA, Johnsen R, Hveem $\mathrm{K}$, et al. The relation between gastroesophageal reflux and respiratory symptoms in a population-based study: the NordTrondelag health survey. Chest. 2006;129(4):1051-6.

28. Schnatz PF, Castell JA, Castell DO. Pulmonary symptoms associated with gastroesophageal reflux: use of ambulatory $\mathrm{pH}$ monitoring to diagnose and to direct therapy. Am J Gastroenterol. 1996;91(9): 1715-8.

29. el-Serag HB, Sonnenberg A. Comorbid occurrence of laryngeal or pulmonary disease with esophagitis in United States military veterans. Gastroenterology. 1997;113(3):755-60.

30. McCarthy DS, Baragar FD, Dhingra S, Sigurdson M, Sutherland JB, Rigby $\mathrm{M}$, et al. The lungs in systemic sclerosis (scleroderma): a review and new information. Semin Arthritis Rheum. 1988;17(4): 271-83.

31. Bouros D, Wells AU, Nicholson AG, Colby TV, Polychronopoulos $\mathrm{V}$, Pantelidis $\mathrm{P}$, et al. Histopathologic subsets of fibrosing alveolitis in patients with systemic sclerosis and their relationship to outcome. Am J Respir Crit Care Med. 2002;165(12):1581-6.

32. Troshinsky MB, Kane GC, Varga J, Cater JR, Fish JE, Jimenez SA, et al. Pulmonary function and gastroesophageal reflux in systemic sclerosis. Ann Intern Med. 1994;121(1):6-10.

33. Denis P, Ducrotte P, Pasquis P, Lefrancois R. Esophageal motility and pulmonary function in progressive systemic sclerosis. Respiration. 1981;42(1):21-4.

34. Marie I, Dominique S, Levesque H, Ducrotte P, Denis P, Hellot MF, et al. Esophageal involvement and pulmonary manifestations in systemic sclerosis. Arthritis Rheum. 2001;45(4):346-54.

35. Savarino E, Bazzica M, Zentilin P, Pohl D, Parodi A, Cittadini G, et al. Gastroesophageal reflux and pulmonary fibrosis in scleroderma: a study using $\mathrm{pH}$-impedance monitoring. Am J Respir Crit Care Med. 2009;179(5):408-13.

36. • Gilson M, Zerkak D, Wipff J, Dusser D, Dinh-Xuan AT, Abitbol V, et al. Prognostic factors for lung function in systemic sclerosis: prospective study of 105 cases. Eur Respir J. 2010;35(1):112-7. This prospective study found that esophageal dysfunction as determined by manometry was not a significant predictor of PFT decline in systemic sclerosis-related ILD.

37. Tobin RW, Pope 2nd CE, Pellegrini CA, Emond MJ, Sillery J, Raghu G. Increased prevalence of gastroesophageal reflux in patients with idiopathic pulmonary fibrosis. Am J Respir Crit Care Med. 1998;158(6):1804-8.

38. Raghu G, Freudenberger TD, Yang S, Curtis JR, Spada C, Hayes J, et al. High prevalence of abnormal acid gastro-oesophageal reflux in idiopathic pulmonary fibrosis. Eur Respir J. 2006;27(1):136-42.

39. Bandeira CD, Rubin AS, Cardoso PF, Moreira Jda S, Machado MM. Prevalence of gastroesophageal reflux disease in patients with idiopathic pulmonary fibrosis. J Bras Pneumol. 2009;35 (12):1182-9.

40. - Fahim A, Dettmar PW, Morice AH, Hart SP. Gastroesophageal reflux and idiopathic pulmonary fibrosis: a prospective study. Medicina (Kaunas). 2011;47(4):200-5. This prospective study revealed that IPF patients do have significant reflux symptoms when evaluated by use of a reflux questionnaire. However, there was no significant difference between EBC pepsin positivity or $H$. pylori infection in IPF patients compared with controls.

41. - Soares RV, Forsythe A, Hogarth K, Sweiss NJ, Noth I, Patti MG. Interstitial lung disease and gastroesophageal reflux disease: key role of esophageal function tests in the diagnosis and treatment. Arq Gastroenterol. 2011;48(2):91-7. This study reestablished that the sensitivity and specificity of GER symptoms alone was very low for ILD and IPF patients. It reveals that esophageal function testing can establish the diagnosis, characterize the esophageal dysfunction, and help in selecting therapy.

42. Sweet MP, Patti MG, Leard LE, Golden JA, Hays SR, Hoopes C, et al. Gastroesophageal reflux in patients with idiopathic pulmonary 
fibrosis referred for lung transplantation. J Thorac Cardiovasc Surg. 2007;133(4):1078-84.

43. D’Ovidio F, Mura M, Tsang M, Waddell TK, Hutcheon MA, Singer LG, et al. Bile acid aspiration and the development of bronchiolitis obliterans after lung transplantation. J Thorac Cardiovasc Surg. 2005;129(5):1144-52.

44. Cantu III E, Appel III JZ, Hartwig MG, Woreta H, Green C, Messier R, et al. J. Maxwell Chamberlain Memorial Paper. Early fundoplication prevents chronic allograft dysfunction in patients with gastroesophageal reflux disease. Ann Thorac Surg. 2004;78(4): 1142-51. discussion 51.

45. Stovold R, Forrest IA, Corris PA, Murphy DM, Smith JA, Decalmer $\mathrm{S}$, et al. Pepsin, a biomarker of gastric aspiration in lung allografts: a putative association with rejection. Am J Respir Crit Care Med. 2007;175(12):1298-303.

46. Collard HR, Moore BB, Flaherty KR, Brown KK, Kaner RJ, King Jr TE, et al. Acute exacerbations of idiopathic pulmonary fibrosis. Am J Respir Crit Care Med. 2007;176(7):636-43.

47. - Lee JS, Song JW, Wolters PJ, Elicker BM, King Jr TE, Kim DS, et al. Bronchoalveolar lavage pepsin in acute exacerbation of idiopathic pulmonary fibrosis. Eur Respir J. 2012;39(2):352-8. This study found significantly elevated pepsin levels in patients experiencing exacerbation of IPF and was the first of its kind to use direct measurement of pepsin in BAL to study the association of IPF exacerbation with reflux disease.

48. - Tcherakian C, Cottin V, Brillet PY, Freynet O, Naggara N, Carton Z, et al. Progression of idiopathic pulmonary fibrosis: lessons from asymmetrical disease. Thorax. 2011;66(3):226-31. This study examined asymmetrical fibrotic patterns on CT scans and found, by objective testing, that those IPF patients had greater symptomatic reflux and GER than patients without asymmetrical disease.
49. • Pillai M, Olson AL, Huie TJ, Solomon JJ, Fernandez-Perez ER, Brown KK, et al. Obstructive sleep apnea does not promote esophageal reflux in fibrosing interstitial lung disease. Respir Med. 2012;106(7):1033-9. This study found that neither OSA nor the apnea hypopnea index (AHI) predicted the presence of GER in patients with fibrosing ILD. Irrespective of body position, GER was no more frequent among those with OSA than among those without OSA.

50. Fein M, Ritter MP, DeMeester TR, Oberg S, Peters JH, Hagen JA, et al. Role of the lower esophageal sphincter and hiatal hernia in the pathogenesis of gastroesophageal reflux disease. J Gastrointest Surg. 1999;3(4):405-10.

51. Linden PA, Gilbert RJ, Yeap BY, Boyle K, Deykin A, Jaklitsch MT, et al. Laparoscopic fundoplication in patients with end-stage lung disease awaiting transplantation. J Thorac Cardiovasc Surg. 2006;131(2):438-46.

52. • Lee JS, Ryu JH, Elicker BM, Lydell CP, Jones KD, Wolters PJ, et al. Gastroesophageal reflux therapy is associated with longer survival in patients with idiopathic pulmonary fibrosis. Am J Respir Crit Care Med. 2011;184(12):1390-4. This large multi-center study demonstrated that use of GER medications is associated with reduced radiographic fibrosis and is an independent predictor of longer survival of IPF patients.

53. •• Lee JS, Collard HR, Anstrom KJ, Martinez FJ, Noth I, et al. Antiacid treatment and disease progression in idiopathic pulmonary fibrosis: an analysis of data from three randomised controlled trials. Lancet. 2013;1(5):369-76. This study analyzed IPF patients included in three large randomized (IPFnet) trials for changes in pulmonary function while taking GERD medication. IPF patients taking antiacid therapy at baseline had a smaller reduction in their FVC than those not taking therapy over time. 